\title{
Expression of testicular angiotensin-converting enzyme in adult spontaneously hypertensive rats
}

\section{Nina Atanassova ${ }^{*}$, Emilia Lakova ${ }^{2 *}$, Yordanka Bratchkova ${ }^{3}$, Genka Krasteva $^{4}$, Miroslav Donchev 5}

${ }^{1}$ Institute of Experimental Morphology and Anthropology with Museum, Bulgarian Academy of Sciences, Sofia, Bulgaria

${ }^{2}$ Department of Biology and Pathophysiology, Medical University, Pleven, Bulgaria

${ }^{3}$ Department of Pathology, Medical University, Pleven, Bulgaria

${ }^{4}$ Department of Physiology and Pharmacology, Medical University, Pleven, Bulgaria

${ }^{5}$ Clinical Center of Nuclear Medicine - Medical University, Pleven, Bulgaria

\begin{abstract}
Recent studies demonstrated that one isoform of angiotensin-converting enzyme named testicular or germinal (tACE) is localized in postmeiotic male germ cells and is essential for fertilizing ability of spermatozoa. Hypertension in spontaneously hypertensive rats (SHR) is androgen-dependent and reduction in male gametes is reported in this experimental conditions. Expression of tACE was not studied under conditions of spontaneous hypertension. The aim of this work is to characterize immunoexpression of tACE in the testis of adult (16-week-old) SHR rats in relation to the changes in blood pressure and serum testosterone level. In $82 \%$ of adult SHR, the immunoexpression of tACE followed the normal stage-specific pattern. Destructive testicular changes, germ cells depletion have been observed in 18\% of 16-week-old SHR and stronger expression of tACE in stages 8-11 compared to controls was detected. As a result stage specificity in SHR was not as evident as in control. No reaction was found in germ cell depleted tubules in which elongated spermatids were absent. Degenerating germ cells exhibited strong immunostaining comparable to that in residual bodies. The blood pressure was significantly higher in SHR and testosterone levels were more than twice but non-significantly elevated. There was no clear correlation between testicular structural changes, blood pressure level values or serum testosterone levels. Expression of tACE in postmeiotic germ cells, specifically altered by SHR, suggested possible involvement of components of reninangiotensin system in the process of spermiogenesis. Loss of enzyme expression we found in germ cell depleted tubules in SHR is due to absence of corresponding stages of spermatid differentiartion. Therefore, tACE can be used as a marker for germ cell depletion due to hypertension and other pathological conditions.
\end{abstract}

Key words: angiotensin-converting enzyme, testis, spermatogenesis, spontaneously hypertensive rats, blood pressure, testosterone

\section{Introduction}

A major part of the experimental hypertension research has been carried out in rats. The spontaneously hypertensive rats (SHR) of Okamoto-Aoki strain were developed by selective breeding of Wistar-Kyoto stock for higher blood pressure [1]. SHRs spontaneously and consistently develop moderate-to-severe hypertension between 7 and 15 weeks of age and have served as model of genetic hypertension in humans. Different

Correspondence: E. Lakova, Dept. of Pathophysiology, Medical University - Pleven 1, St. Kliment Ochridski Str. 5800 Pleven, Bulgaria; e-mail: elakova@yahoo.co.uk mechanisms contributing to higher blood pressure in SHR have been elucidated. Hypertension in SHR is androgen-dependent, as in many other genetic and experimentally induced models of hypertension [2]. However, some reports provide evidence about activation in hypothalamo-pituitary-testicular axis in male SHR $[3,4]$. On the other hand, a severe reduction in the number of mature and immature male gametes has been described in SP-SHR (SHR substrain predisposed to cerebrovascular complications). The structural hypertensive changes in intratesticular arterioles, as well as in

\footnotetext{
* N.A. and E.L. contributed equally to this study and should be considered as joint first authors.
} 
different vascular beds of SP-SHR, have been suggested to cause damage of spermatogenesis $[5,6]$.

Angiotensin-converting enzyme (ACE), well-known component of renin-angiotensin system, exists as two isoforms - somatic and testicular. Somatic ACE (sACE) is responsible for the conversion of angiotensin I to the potent vasoconstrictor angiotensin II (Ang II) and inactivation of both vasodilator peptides - bradykinin and angiotensin-(1-7). Thus, sACE has been implicated in the control of blood pressure and fluid-electrolyte balance. In the male reproductive system sACE has been localized in testicular Leydig cells, epididymal epithelial cells, epididymal fat pad, and prostatic glandular epithelium [7]. Ang II locally produced by sACE could modulate steroidogenesis in Leydig cells, regulates secretory function of epididymis, plays role in anion secretion, tubular contractility and enhancement of sympathetic nerve activity in the prostate. Ang II has been shown to maintain sperm motility and to stimulate capacitation.

The testis-specific isozyme, termed testicular or germinal ACE (tACE), is encoded by the ACE gene and raised from alternate transcription initiation. Testicular ACE is structurally related with SACE - both isoforms share completely homologous $\mathrm{C}$-domain. This isoform of ACE is expressed only in spermatids and spermatozoa and is testosterone dependent [8]. The substrate preference of tACE is still unknown and this isoenzyme does not generate Ang II in vivo. Testicular ACE has been shown to play an essential role in the control of the male reproductive system[9] . Recently, dipeptidase activity of tACE was identified as factor of sperm-zona pellucida binding [10]. On the other hand, tACE acts as releasing factor for some proteins (Tesp5 and $\mathrm{Ph}-20$ ) anchored on spermatozoa membrane and necessary for fertilization [11]. Based on these findings, it can be suggested that tACE may serve as marker of fertilizing ability of spermatozoa.

The role of tACE and its localization in the testis is not suited in conditions of spontaneous hypertension. In this respect the current study was undertaken to characterize immunoexpression of tACE in the testis of SHR rats in relation to the changes in blood pressure and serum testosterone level.

\section{Materials and methods}

Animals. The experiments were carried out on 11 male spontaneously hypertensive rats (SHR) (Okamoto-Aoki strain) of age 16 weeks. Age-and sex-matched normotensive Wistar rats $(n=6)$ served as controls. The rats were housed under standard conditions, food and tap water were provided ad libitum. The study was performed in accordance with Institutional care and use of laboratory animal guidelines, Medical University, Pleven.

Measurement of arterial blood pressure indirectly in conscious rat. Systolic blood pressure (SBP) was measured in conscious rats that were restrained, by means of the tail-cuff method using sphygmomanometer (Ugo Basile-58000, Italy). Prior to measuring SBP the rats were warmed at $37^{\circ} \mathrm{C}$ for $15 \mathrm{~min}$. Levels of SBP were determined by averaging 3 values recorded.

Tissue and blood collection. After body weight was recorded for each animal, the abdominal cavity was opened under urethan anesthesia $(780 \mathrm{mg} / \mathrm{kg}$, i.p.). Blood was then collected from the bifurcation of the aorta. The serum was separated and frozen at $-20^{\circ} \mathrm{C}$ until assay. Subsequently to blood collection, one testis per animal was removed and weighed. Relative weights of testes were presented as $\mathrm{g} / 100 \mathrm{~g}$ body weight.

Immunohistochemistry for tACE. Testicular tissue was fixed by immersion in freshly prepared Bouin's solution for 24 hours at room temperature and routinely processed for embedding in paraffin. For immunohistochemical analysis, $5-\mu \mathrm{m}$-thick sections were cut and mounted on silanized slides. Following dewax in xylene and rehydration in graded alcohol solutions, the sections were subjected to antigen retrieval in $0.01 \mathrm{M}$ Citrate buffer, $\mathrm{pH}=6.0$ at $95^{\circ} \mathrm{C}$ for $5 \mathrm{~min}$ water bath. Then slides were incubated in $3 \%(\mathrm{v} / \mathrm{v})$ hydrogen peroxide in methanol for $5 \mathrm{~min}$ at room temperature to block endogenous peroxidase followed by 2 washes in PBS for 5 minutes each. Tissue sections were blocked for 1 hour in $1.5 \%$ dunky blocking serum in PBS. Primary antibody ACE (sc- 12187, Santa Cruz Biotechnology,Inc. Santa Cruz, Calif, USA) diluted 1:500 was dropped onto the sections for $30 \mathrm{~min}$ incubation in humidified chamber at $37^{\circ} \mathrm{C}$. After 3 washes PBS (5 minutes each) goat biotinylated secondary antibody-ABC staining system (sc-2023, Santa Cruz Biotechnology, Inc. Santa Cruz, Calif, USA) was applied and liquid DAB (K3468, DAKO, Glostrup, Denmark) was used as chromogen. Sections were counterstained with hematoxylin (5 min) and after dehydration they are mounted in Entellan mounting media (1.07961. Merck, KGaA, Darmstadt, Germany). Testicular ACE immunoreactivity was identified as brown staining. Negative controls were run routinely in parallel by omitting of the primary antibody. Sections were analyzed and photographed using Zeiss Axiostar-plus microscope and digital camera Sonny DSC-S75.

Hormonal analysis. The serum total testosterone levels were analyzed using commercially available radioimmunoassay kit (catalogue no: KIP 1709, BioSource Europe SA, Belgium). The limit of sensitivity was $0.025 \mathrm{ng} / \mathrm{ml}$.The intra- and interassay coefficients of variation were 14.8 and $15 \%$, respectively.

Statistical analysis. Results are expressed as mean \pm SEM and were analyzed for statistically significant differences using oneway ANOVA, followed by t-test. Statistical significance was accepted at value of $\mathrm{p}<0.05$

\section{Results}

\section{Body and testis weight}

The absolute testicular weight was not statistically different between control rats and SHRs. The SHRs showed higher relative testicular weight evaluated as testis weight/body weight (TBW) ratio than Wistar rats. There was statistically significant difference in the body weight between SHR and control animals (Table 1).

\section{Blood pressure}

The systolic blood pressure of the male SHR at age 16 weeks was significantly higher than in age-and sexmatched control normotensive Wistar rats, as shown in Table 1. 
Table 1. Systolic blood pressure, body weights, absolute and relative testicular weights, and serum testosterone levels in SHR and normotensive control Wistar rats. Values are expressed as means \pm SEM.

\begin{tabular}{|l|c|c|c|}
\hline \multicolumn{1}{|c|}{ Lind points } & Wistar rals $(\mathrm{n}=6)$ & SIIR $(\mathrm{n}=1 \mathrm{l})$ & $\mathrm{p}$ \\
\hline $\mathrm{RR}(\mathrm{mm} \mathrm{Ilg})$ & $126.667 \pm 2.472$ & $176.364 \pm 1.521$ & $\mathrm{p}<0.00 \mathrm{l}$ \\
\hline $\mathrm{BW}(\mathrm{g})$ & $401.667 \pm 15.365$ & $251.364 \pm 5.397$ & $\mathrm{p}<0.001$ \\
\hline Testis $(\mathrm{g})$ & $1.460 \pm 0.102$ & $1.404 \pm 0.101$ & $\mathrm{p}>0.05$ \\
\hline Index Testis $/ \mathrm{BW}(\mathrm{g} / 100 \mathrm{~g})$ & $0.367 \pm 0.029$ & $0.563 \pm 0.043$ & $\mathrm{p}<0.01$ \\
\hline Testosterone $(\mathrm{ng} / \mathrm{ml})$ & $1.425 \pm 0.434$ & $3.15 \pm 0.741$ & $\mathrm{p}>0.05$ \\
\hline
\end{tabular}

Table 2. Semiquantitative evaluation of tACE immunoexpression at the stages of the seminiferous epithelium and steps of spermiogenesis in normotensive control and SHR adult rats.

\begin{tabular}{|c|c|c|c|c|c|c|c|c|c|}
\hline \multirow{3}{*}{$\begin{array}{c}\text { Exp. } \\
\text { Group }\end{array}$} & \multicolumn{9}{|c|}{ Stages of the seminiferous epithelium } \\
\cline { 2 - 10 } & \multicolumn{9}{|c|}{ Steps of spermiogenesis } \\
\cline { 2 - 10 } & \multicolumn{2}{|c|}{ VII } & \multicolumn{2}{|c|}{ VIII } & IX & X-XI & XII-XIV & I-III & IV-VI \\
\cline { 2 - 10 } & 7 & 19 & 8 & 19 & 9 & $10-11$ & $12-14$ & $15-16$ & $17-18$ \\
\hline Control & - & ++++ & $-/+$ & ++++ & + & ++ & ++++++ & +++ & ++++ \\
\hline SIIR & $-/+$ & ++++ & + & ++++ & $+/++$ & +++ & ++++ & ++++ & ++++ \\
\hline
\end{tabular}

\section{Immunoexpression of $t A C E$}

Immunohistochemical analysis revealed stage-specific pattern of tACE expression in postmeiotic germ cells. First faint immunoreactivity appeared in the cytoplasm of round spermatids step 8 in a round shape manner (Fig. 1A, Table 2). Weak intensity was found in elongating spermatids step 9 at stage IX of the cycle of seminiferous epithelium (Fig. 1B). Late that stage the immunostaining progressively increased and was located in caudally organized cytoplasm of elongating spermatids. Medium intensity of reaction was observed in spermatids step10-11 at stages X-XI of the cycle of seminiferous epithelium (Fig. 1A, B). Immunoexpression became strong later than steps 12 of spermiogenesis (stage XII of the cycle; Fig. 1B, C) and reached maximum in steps 17-19 stages (IV-VIII of the cycle; Fig. 1D). Strong immunoreactivity was confined to residual bodies that were numerous in the lumen of seminiferous tubules at stages VIII-IX of the cycle (Fig. 1A, B). Residual bodies were also found in the basal part of the tubules where they were phagocytosed by Sertoli cells. Flagela of late elongated spermatids step 18-19 were also strongly reactive. No immunoexpression was observed in other germ cell types (spermatogonia, spermatocytes) as well as in somatic cells (peritubular cells, Leydig and Sertoli cells).

Most of the experimental animals in SHR group revealed intact testicular morphology accompanied by increased relative testis weigh (testis/body weight ratio). Expression of tACE occurred in normal stage-specific pattern like in controls. In $18 \%$ of SHR rats profound destructive changes were seen in testicular histology manifested by germ cell depletion, irregular shape and reduced diameter of seminiferous tubules. In these animals relative testis weight were normal or reduced. With some exceptions, the stage specificity of tACE expression broadly followed the normal pattern. First immunoreactivity appeared in the cytoplasm of round spermatids step VII being more distinguishable in stage VIII compared to controls (Fig. 1E, Table 2). Cellular membranes of round spermatids were strongly reactive that was not found in control rats. Immunoexpression of tACE in spermatids 9-14 were more intensive than corresponding stages in the controls. As a result stage-specificity, in particular, gradual increase in reaction intensity among stages was not as obvious as in control animals. Germ cell depletion was manifested by loss of elongating and round spermatids and in most severe cases by lack of spermatocytes. Sertoli cell only tubules (SCO) containing only Sertoli cells and few spermatogonia were also observed. No reaction was found in germ cell depleted tubules in which elongated spermatids step 819 were absent (Fig. 1F). Degenerating germ cells exhibited strong immunostaining comparable to that in residual bodies (Fig. 1G)

\section{Serum testosterone levels}

Serum testosterone concentration in the SHR showed more than 2 time increase compared to the control although the difference was not statistically significant (Table 1). 

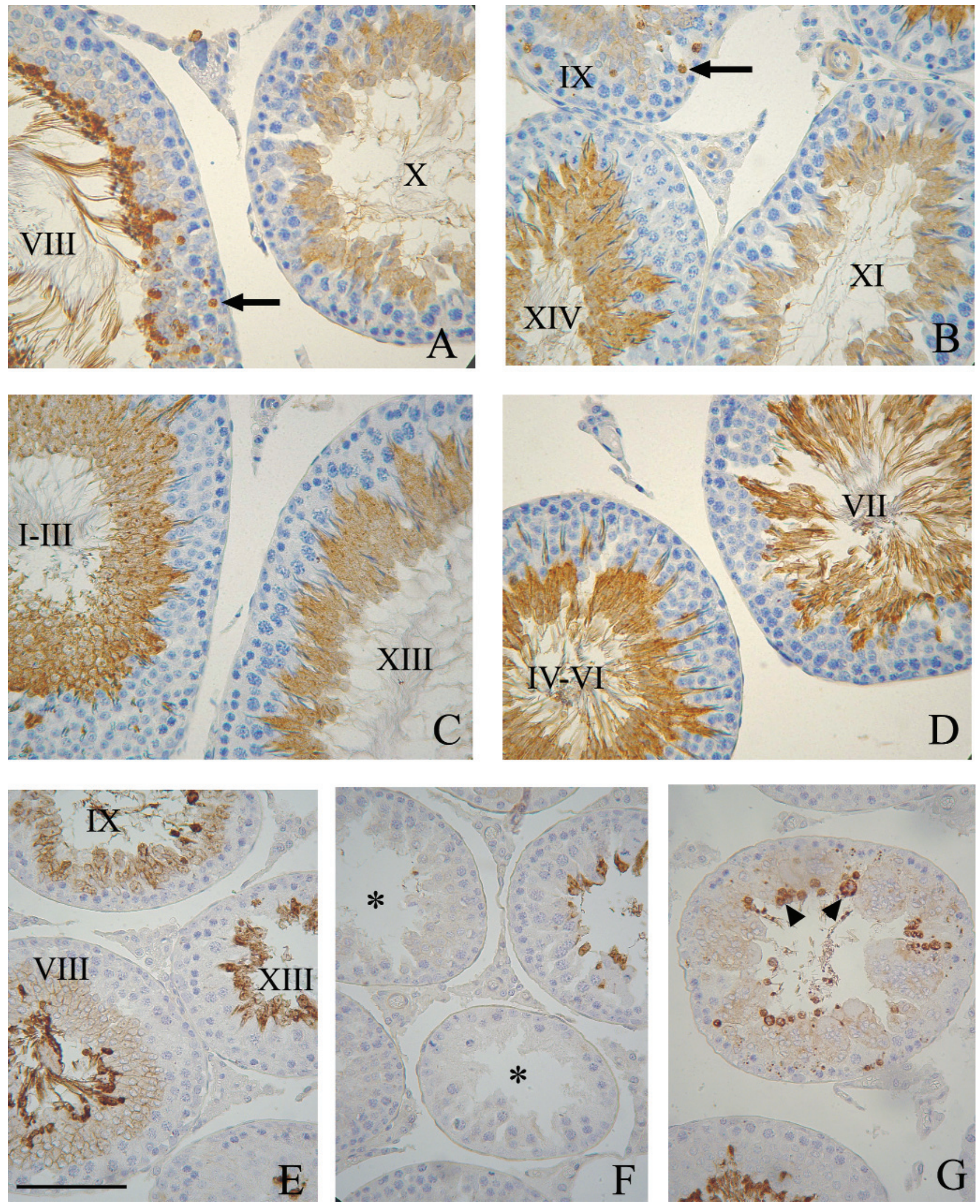

Fig. 1. Stage-specific immunoexpression of tACE in the cytoplasm of differentiating spermatids in adult control (A-D) and hypertensive (E-G) rats. Note the minimal intensity at step 8 and maximal intensity at step 19 of spermiogenesis, both at stage VIII of the spermatogenic cycle. E. Note stronger immunoreactivity in SHR at stages IX-XIII compared to the same stages in control (A-C); F. Lack of ACE expression in germ cell depleted tubules (asterisk) with absence of elongated spermatids step 8-19; G. Strong immunostaining of degenerating germ cells (arrowheads) comparable to that in residual bodies (arrows). Scale bar $-50 \mu \mathrm{m}$. 


\section{Discussion}

In the present study, we focused on expression of tACE in the testis having in mind the essential role of the enzyme for sperm quality. Recently, quantification of tACE on the surface of humane spermatozoa has been performed by flow cytometry and immunohistochemistry, using a set of highly specific monoclonal antibodies recognizing the testicular isoform of ACE [12]. The highest concentrations of tACE have been reported on spermatozoa with normal morphological characreristics and motility [13]. Moreover, positive correlation exists between spermatozoon movement and expression of tACE [14]. Thus, tACE expression on human spermatozoa is of clinical importance for the diagnosis of some forms of male infertility. Based on polymorphisms in tACE -gene in infertile men, some authors did not find any association between tACE and male fertility [15].

Besides the clinical studies, experimental research in transgenic mice reveals more details about physiological role of tACE. For example, ACE-null mice exibited reduced blood pressure, renal atrophy, renal vascular hyperplasia, anemia and male sterility as a result of the absence of both ACE isoforms- SACE and tACE. The fertility defect was due to poor sperm migration through the oviduct and failure to bind and penetrate the zona pellucida [16]. The expression of transgenic sACE exclusively in vascular endothelial cells of ACE-null mice restores blood pressure, but male mice remain sterile, indicated that $\mathrm{sACE}$ can not substitute for tACE in supporting male fertility [17].

The present study demonstrated stage-specific expression of tACE in the cytoplasm of male germ cells of 16-week-old normal Wistar rats. Gradual increase of immunostaining was evident from step 8 to step 19 of spermiogenesis. With one exception our results are consistent with data by Sibony et al [18] in 8-10 week-old Sprague-Dawley rats. Discrepancy is related to weaker immunoreactivity in elongating spermatids at steps 15 17 compared to earlier steps. As a result gradual increased immunoexpression of tACE during spermiogenesis is not observed. In another study by Langford et al. [19] tACE immunoreactivity in mouse testis was detected later than step 10 spermatids. The differences between our data and those by both author groups could be explained by using different antibodies against the portion common to the testicular and somatic ACE isoforms. Species-specific expression of tACE was demonstrated in human testis where reaction was found only in adluminal membranes of postmeiotic germ cells later than step 3 round spermatids [20] corresponding to step 7 round spermatids in rat.

In the present study we provide new data about immunoexpression of tACE in testes of adult SHR at earlier step of spermiogenesis compared with agematched control normotensive Wistar rats. By comparison of corresponding stages, more intensive immunos- taining of tACE in adult SHR has been described than controls. This finding could be a consequence of testosterone effects in SHR, as tACE is under androgen control [8]. In our study testosterone levels were elevated in immature and adult SHR animals. Significantly higher testosterone levels were detected in younger group of SHR (6 weeks of age, data not shown). More than twice but non-significant increase of testosterone concentrations in adult SHR rats was due to highly variable values between animals within SHR group. Thus, high androgen exposure in earlier age might explain changes of tACE expression found later in adulthood.

Destructive testicular changes and germ cells depletion have been observed in our study in 18\% (2 of 11 rats) of adult SHR rats. There was no clear correlation between degree of testicular damage, blood pressure values, and serum testosterone level. A possible causative role of vascular changes in SHR for disorders in seminiferous epithelium could be discussed. Several reports described structural and functional alterations in arterial vessels of some vascular beds (e.g. mesenteric arteries) not only in adult SHR rats [21], but in younger (3-4-week-old) SHR animals in prehypertensive stage [22]. Similarly, vascular changes affected the structure and function of erectile tissue in SHR underlying erectile dysfunction in prehypertensive SHR [23]. Moreover, in SP-SHR rats (severe form of hypertension) the structural changes in testicular arterioles appeared early at 15 weeks of age and progressed rapidly [5]; afterwards manifested by more severe alterations in testicular arteries [24] and seminiferous epithelium (atrophic tubules, significant reduction in number of round spermatids) [5]. A possible mechanism of testicular damage involves ischemia due to vascular changes, reduced nutrition supply, Sertoli cells dysfunction. Improved spermatogenesis in SP-SHR was reported after therapy of vascular changes by calcium antagonist minidipine [25]. It become evident that severe testicular atrophy occurred later in life of hypertensive animals, especially in SP-SHR where high values of blood pressure were measured and severe vascular damage was detected. This could explain why most of the SHRs in our experiment (82\%) exhibited normal testicular morphology associated with short-term mild hypertension.

Stage specificity of tACE localization during spermatogenic cycle characterizes tACE as a good marker for stages of spermatid differentiation. Expression of tACE starts and reaches maximum in androgen dependent stage VIII of the spermatogenic cycle that implies androgen regulation of enzyme production in postmeiotic germ cells. In this respect, future investigations involving proper experimental models for androgen ablation is needed and such studies are in progress by our group.

Expression of tACE in postmeiotic germ cells, specifically altered by SHR, suggested possible involvement of 
components of renin-angiotensin system in the process of spermiogenesis. Our previous study demonstrated an important role of elements of kallikrein-kinin system, in particular bradikynin, for male germ cell proliferation and hence for regulation of germ cell number [26]. Loss of enzyme expression we found in germ cell depleted tubules in SHR is due to absence of corresponding stages of spermatid differentiartion. Therefore, tACE can be used as a marker for the degree of germ cell depletion due to hypertension and other pathological conditions.

In conclusion, to date, there is a lack of compelling data specifically linking hypertension with impairment of male reproductive potential. In this regard our study provides useful information about relationship between hypertension, disturbance of spermatogenesis and androgen production. Not only severe and longterm hypertension, but moderate and short-term hypertension could be also a risk factor for male reproductive health.

Acknowledgements: We thank Mrs Tereza Dineva and Mr Valeri Ivanov for technical assistance. This study was supported by a grant from Medical University of Pleven.

\section{References}

[ 1] Okamoto K, Aoki K. Development of strain of spontaneously hypertensive rats. Jpn Circ J. 1963;27:282-293.

[2] Reckelhoff JF, Zhang H, Srivastava K, Granger JP. Gender differences in hypertension in spontaneously hypertensive rats: role of androgens and androgen receptor. Hypertension. 1999;34:920-923.

[ 3] Rodriguez-Padilla ML, Bellido C, Pinilla L, Aguilar E. Secretion of LH in spontaneously hypertensive rats. $J$ Endocrinol. 1987;113:255-260.

[4] Aguilar E, Rodriguez-Padilla ML, Bellido C, Tena-Sempere M, Pinilla L.Changes in follicle-stimulating hormone secretion in spontaneously hypertensive rats. Neuroendocrinology. 1992; 56:85-93.

[5] Akagashi K, Itoh N, Kumamoto Y, Tsukamoto T, Suzuki Ts, Ohta Y. Hypertensive changes in intratesticular arteries impair spermatogenesis of the stroke-prone spontaneously hypertensive rat. J Androl. 1996;17:367- 374

[6] Itoh N, Akagashi K, Kumamoto Y, Suzuki T, Ohta Y. Influence of hypertensive vascular changes in intratesticular arteries on spermatogenesis in SHRSP. Clin Exp Pharmacol Physiol Suppl. 1995;22:S134-S135.

[ 7] Leung PS, Sernia C. The renin-angiotensin system and male reproduction: new functions for old hormones. $J \mathrm{Mol}$ Endocrinol. 2003;30:263-270.

[ 8] Velletri PA, Aquilano DR, Bruckwick E, Tsai-Morris CH, Dufau ML, Lovenberg W. Endocrinological control and cellular localization of rat testicular angiotensin-converting enzyme (EC 3.4.15.1). Endocrinology. 1985;116:2516-2522.

[ 9] Hagaman JR, Moyer JS, Bachman ES, et al. Angiotensin-converting enzyme and male fertility. PNAS. 1998;95:2552-2557.

[10] Deguchi E, Tani T, Watanabe H, Yamada Sh, Kondoh G. Dipeptidase- inactivated tACE action in vivo: selective inhibition of sperm-zona pellucida binding in the mouse. Biol Reprod. 2007;77:794-802.
[11] Kondoh G, Tojo H, Nakatani Y, et al. Angiotensin-converting enzyme is a GPI-anchored protein releasing factor crucial for fertilization. Nature Medicine. 2005;11:60 -166.

[12] Nikolaeva MA, Balyasnikova IV, Alexinskaya MA, et al. Testicular isoform of angiotensin I-converting enzyme (ACE,CD143) on the surface of human spermatozoa: revelation and quantification using monoclonal antibodies. $A m \mathrm{~J}$ Reprod Immunol. 2006;55:54-68.

[13] Aleksinskaya MA, Nikolaeva MA, Danilov SM, Elistratova OS, Sukhikh GT. Quantitative study of testicular angiotensinconverting enzyme on the surface of human spermatozoa. Bull Exp Biol Med. 2006;141:41-44.

[14] Aleksinskaya MA, Nikolaeva MA, Danilov SM, et al. Relationship between spermatozoon movement velocity and expression of testicular isoform of angiotensin-converting enzyme on their surface. Bull Exp Biol Med. 2006;141:198201.

[15] Liao WX, Roy AC. Lack of association between polymorphisms in the testis-specific angiotensin converting enzyme gene and male infertility in an Asian population. Mol Hum Reprod. 2002;8:299-303.

[16] Esther CR, Howard TE, Marino E M, Goddard J. M, Capecchi MR, Bernstein KE. Mice lacking angiotensin-converting enzyme have low blood pressure, renal pathology, and reduced male fertility. Lab Invest. 1996;74:953-965.

[17] Kessler SP, Rowe TM, Gomos JB, Kessler PM, Sen GC. Physiological non-equivalence of the two isoforms of angiotensin-converting enzyme. J Biol Chem. 2000;275: 26259-26264.

[18] Sibony M, Segretain D, Gasc JM. Angiotensin-converting enzyme in murine testis: step-specific expression of the germinal isoform during spermiogenesis. Biol Reprod. 1994;50: 1015-1026.

[19] Langford KG, Zhou Y, Russell LD, Wilcox JN, Bernstein KE. Regulated expression of testis angiotensin-converting enzyme during spermatogenesis in mice. Biol Reprod. 1993; 48:12101218.

[20] Pauls K, Metzger R, Steger K, Klonisch T, Danilov S, Franke FE. Isoforms of angiotensin I-converting enzyme in the development and differentiation of human testis and epididymis. Andrologia. 2003;35:32-43.

[21] Inoue T, Masuda T, Kishi K. Structural and functional alterations of mesenteric vascular beds in spontaneously hypertensive rats. Jpn Heart J. 1990;31:393-403.

[22] Dickhout JG, Lee RMKW. Structural and functional analysis of small arteries from young spontaneously hypertensive rats. Hypertension. 1997;29:781-789.

[23] Behr-Roussel D, Gorny D, Mevel K, et al. Erectile dysfunction: an early marker for hypertension? A longitudinal study in spontaneously hypertensive rats. Am J Physiol Regul Integr Comp Physiol. 2005;288:R276-R283.

[24] Saito N, Kawamura H. The incidence and development of periarteritis nodosa in testicular arteries and mesenteric arteries of spontaneously hypertensive rats. Hypertens Res. 1999;22:105-112.

[25] Akagashi K, Kumamoto Y, Itoh N, Tsukamoto T, Suzuki TS, Ohta Y. Minidipine improves spermatogenesis in the strokeprone spontaneously hypertensive rat. J Androl. 1997;18: $210-216$

[26] Atanassova N. B2 bradykinin receptor mediates the stimulatory effect of bardykinin on rat germ cell proliferation in vitro. Andrologia. 2003;35:13-16.

Submitted: 15 August, 2008 Accepted after reviews: 26 September, 2008 